PROCEEDINGS OF THE

AMERICAN MATHEMATICAL SOCIETY

Volume 133, Number 7 , Pages 2077-2081

S 0002-9939(05)07722-1

Article electronically published on January 31, 2005

\title{
AN INTERPOLATION THEOREM FOR HILBERT SPACES WITH NEVANLINNA-PICK KERNEL
}

\author{
BJARTE BØE
}

(Communicated by Juha M. Heinonen)

\begin{abstract}
We prove an interpolation theorem for Hilbert spaces of analytic functions that have the Nevanlinna-Pick property. This result applies to Dirichlet and Dirichlet-type spaces, and in particular a short proof of the theorem by Marshall-Sundberg on interpolating sequences is obtained.
\end{abstract}

\section{INTRODUCTION}

We consider Hilbert spaces of analytic functions in the unit disk with inner product

$$
\langle f, g\rangle=\sum_{n} \frac{1}{c_{n}} a_{n} \overline{b_{n}},
$$

where $f(z)=\sum_{n} a_{n} z^{n}, g(z)=\sum_{n} b_{n} z^{n}$. The reproducing kernels $k_{w}(z)$ satisfying

$$
\left\langle f, k_{w}\right\rangle=f(w)
$$

are given by $k_{w}(z)=k(\bar{w} z)$, where

$$
k(z)=\sum_{n} c_{n} z^{n}
$$

We will assume that $k$ satisfies

$$
1-\frac{1}{k(z)}=\sum_{n} d_{n} z^{n}
$$

with $d_{n} \geq 0$ and $k(r)>0$ for $r>0$. This holds e.g. for Dirichlet and Dirichlet-type spaces ([6, p. 22]).

A Hilbert space $H$ has the Nevanlinna-Pick property when the matrix

$$
\left(1-w_{i} \bar{w}_{j}\right)\left\langle k_{z_{i}}, k_{z_{j}}\right\rangle
$$

being positive semi-definite is necessary and sufficient for the existence of $\phi$ in the multiplier algebra $M_{H}$ of $H$ satisfying $\phi\left(z_{i}\right)=w_{i},\|\phi\|_{M_{H}} \leq 1$. In [6] Lemma 10] it is proved that (1) implies the Nevanlinna-Pick property.

We define $\left\{z_{n}\right\}$ to be interpolating for $H$ when

$$
\left\{\frac{f\left(z_{n}\right)}{\left\|k_{z_{n}}\right\|_{H}}: f \in H\right\}=l^{2}
$$

Received by the editors September 30, 2003 and, in revised form, March 12, 2004.

2000 Mathematics Subject Classification. Primary 30H05, 46E22.

(C)2005 American Mathematical Society 
and to be interpolating for $M_{H}$ when

$$
\left\{\phi\left(z_{n}\right): \phi \in M_{H}\right\}=l^{\infty} .
$$

Also, $\left\{\frac{k_{z_{n}}}{\left\|k_{z_{n}}\right\|_{H}}\right\}$ is a Riesz sequence if

$$
\left\|\sum_{n} a_{n} \frac{k_{z_{n}}}{\left\|k_{z_{n}}\right\|_{H}}\right\| \sim\left(\sum_{n}\left|a_{n}\right|^{2}\right)^{\frac{1}{2}} .
$$

For spaces with the Nevanlinna-Pick property, these three conditions are satisfied by the same sequences (6, Corollary 7], [2, Theorem 9.19]). Our main result is a characterisation of these sequences under an extra assumption. To state this, we say $\left\{z_{n}\right\}$ is separated when

$$
\left|\left\langle\frac{k_{z_{n}}}{\left\|k_{z_{n}}\right\|}, \frac{k_{z_{m}}}{\left\|k_{z_{m}}\right\|}\right\rangle\right|<\sigma
$$

for $n \neq m, \sigma<1$. We also make the further technical assumption that for any sequence $\left\{z_{n}\right\}$, boundedness on $l^{2}$ of the Gram matrix

$$
\left\{\left\langle\frac{k_{z_{n}}}{\left\|k_{z_{n}}\right\|}, \frac{k_{z_{m}}}{\left\|k_{z_{m}}\right\|}\right\rangle\right\}
$$

implies boundedness of the matrix

$$
\left\{\left|\left\langle\frac{k_{z_{n}}}{\left\|k_{z_{n}}\right\|}, \frac{k_{z_{m}}}{\left\|k_{z_{m}}\right\|}\right\rangle\right|\right\} .
$$

Under these assumptions we have the following:

Theorem 1.1. Suppose $H$ is a Hilbert space of analytic functions satisfying (1) and the condition on the Gram matrix. A sequence $\left\{z_{n}\right\}$ is interpolating for $H$ if and only if it is separated and the Gram matrix is bounded.

The assumptions in Theorem 1.1 can be stated in a way that looks more concrete. For this, define $\mu$ to be a Carleson measure for $H$ if

$$
\int|f|^{2} d \mu \lesssim\|f\|^{2}
$$

Boundedness of the Gram matrix is equivalent to $\sum_{n}\left\|k_{z_{n}}\right\|^{-2} \delta_{z_{n}}$ being a Carleson measure ([2, Proposition 9.5]).

As a convenient reference for most results that we use, we mention [2], especially chapters 7 and 9 .

\section{Proof of Theorem 1.1}

It is clear that an interpolating sequence must have a bounded Gram matrix. The separation condition is proved as in [6, p. 41].

For the converse we will prove that $\left\{\frac{k_{z_{n}}}{\left\|k_{z_{n}}\right\|_{H}}\right\}$ is a Riesz sequence. According to Bari's theorem ([7, p. 132]), this holds if and only if both matrices

$$
\Gamma=\left\langle\frac{k_{z_{n}}}{\left\|k_{z_{n}}\right\|}, \frac{k_{z_{m}}}{\left\|k_{z_{m}}\right\|}\right\rangle, \Gamma^{\prime}=\left\langle f_{n}, f_{m}\right\rangle
$$

are bounded operators on $l^{2}$. Here $\left\{f_{n}\right\}$ are biorthogonal functionals defined as minimal norm solutions of

$$
\left\langle f_{n}, \frac{k_{z_{m}}}{\left\|k_{z_{m}}\right\|}\right\rangle=\delta_{n, m} .
$$


It is clear that $\left\{f_{n}\right\}$ lie in the span of $\left\{k_{z_{n}}\right\}$.

For $A \subset\left\{z_{i}\right\}$, denote by $H_{A}$ the Hilbert space $\left\{f \in H: f_{\mid A}=0\right\}$ and by $k_{\alpha}^{A}$ the reproducing kernel for $H_{A}$ at $\alpha$. It satisfies

$$
k_{\alpha}^{A}(\alpha)=\left\|k_{\alpha}^{A}\right\|_{H_{A}}^{2}
$$

and maximises $|f(\alpha)|$ among $f$ in $H_{A}$ with $\|f\|=\left\|k_{\alpha}^{A}\right\|$. So we have

$$
f_{n}=\frac{k_{z_{n}}^{A_{n}}}{\left\|k_{z_{n}}^{A_{n}}\right\|^{2}} \cdot\left\|k_{z_{n}}\right\|
$$

where $A_{n}=\left\{z_{i}\right\} \backslash z_{n}$.

To prove $\Gamma^{\prime}$ is bounded on $l^{2}$ we write

$$
\left\langle f_{n}, f_{m}\right\rangle=\frac{\left\|k_{z_{n}}\right\|\left\|k_{z_{m}}\right\|}{\left\|k_{z_{n}}^{A_{n}}\right\|^{2}\left\|k_{z_{m}}^{A_{m}}\right\|^{2}}\left\langle k_{z_{n}}^{A_{n}}, k_{z_{m}}^{A_{m}}\right\rangle .
$$

Reproducing kernels for $H_{A \cup a}$ are determined by those for $H_{A}$ by the relation

$$
k_{\alpha}^{A \cup a}(z)=k_{\alpha}^{A}(z)-\frac{k_{a}^{A}(z) k_{\alpha}^{A}(a)}{k_{a}^{A}(a)} .
$$

Denoting $A^{\prime}=\left\{z_{i}\right\} \backslash\left\{z_{n} \cup z_{m}\right\}$, we have

$$
k_{z_{n}}^{A_{n}}(z)=k_{z_{n}}^{A^{\prime}}(z)-\frac{k_{z_{m}}^{A^{\prime}}(z) k_{z_{n}}^{A^{\prime}}\left(z_{m}\right)}{k_{z_{m}}^{A^{\prime}}\left(z_{m}\right)}
$$

and

$$
k_{z_{m}}^{A_{m}}(z)=k_{z_{m}}^{A^{\prime}}(z)-\frac{k_{z_{n}}^{A^{\prime}}(z) k_{z_{m}}^{A^{\prime}}\left(z_{n}\right)}{k_{z_{n}}^{A^{\prime}}\left(z_{n}\right)} .
$$

We get from (4)

$$
\begin{array}{r}
\left\langle k_{z_{n}}^{A_{n}}, k_{z_{m}}^{A_{m}}\right\rangle=\left\langle k_{z_{n}}^{A_{n}}, k_{z_{m}}^{A^{\prime}}\right\rangle-\frac{k_{z_{m}}^{A^{\prime}}\left(z_{n}\right)}{k_{z_{n}}^{A^{\prime}}\left(z_{n}\right)}\left\langle k_{z_{n}}^{A_{n}}, k_{z_{n}}^{A^{\prime}}\right\rangle \\
=k_{z_{n}}^{A_{n}}\left(z_{m}\right)-\frac{k_{z_{m}}^{A^{\prime}}\left(z_{n}\right)}{k_{z_{n}}^{A^{\prime}}\left(z_{n}\right)} k_{z_{n}}^{A_{n}}\left(z_{n}\right) .
\end{array}
$$

From (3) it follows that $k_{z_{n}}^{A_{n}}\left(z_{n}\right) \leq k_{z_{n}}^{A^{\prime}}\left(z_{n}\right)$, so

$$
\left|\left\langle k_{z_{n}}^{A_{n}}, k_{z_{m}}^{A_{m}}\right\rangle\right| \leq\left|k_{z_{m}}^{A^{\prime}}\left(z_{n}\right)\right| .
$$

Referring to (2) we then have

$$
\left|\left\langle f_{n}, f_{m}\right\rangle\right| \leq \frac{\left\|k_{z_{n}}\right\|\left\|k_{z_{m}}\right\|}{\left\|k_{z_{n}}^{A_{n}}\right\|^{2}\left\|k_{z_{m}}^{A_{m}}\right\|^{2}}\left|k_{z_{m}}^{A^{\prime}}\left(z_{n}\right)\right| .
$$

For $A \subset\left\{z_{i}\right\}, z_{j} \notin A$, the extremal problems

$$
c_{H}=\inf \left\{\|f\|_{H}: f\left(z_{j}\right)=1, f_{\mid A}=0\right\}
$$

and

are related by

$$
c_{M_{H}}=\inf \left\{\|\phi\|_{M_{H}}: \phi\left(z_{j}\right)=1, \phi_{\mid A}=0\right\}
$$

$$
c_{M_{H}}=c_{H}\left\|k_{z_{j}}\right\|
$$


and the unique extremals satisfy

$$
f_{z_{j}}=\phi_{z_{j}} \frac{k_{z_{j}}}{\left\|k_{z_{j}}\right\|^{2}} .
$$

This follows from the Nevanlinna-Pick property [6, Proposition 6]. In [6, Proposition 23] and [9] it is proved that

$$
c_{M_{H}} \leq \prod_{z_{i} \in A}\left(1-\frac{\left|\left\langle k_{z_{i}}, k_{z_{j}}\right\rangle\right|^{2}}{\left\|k_{z_{i}}\right\|^{2}\left\|k_{z_{j}}\right\|^{2}}\right) .
$$

By the Carleson condition applied to $\frac{k_{z_{j}}}{\left\|k_{z_{j}}\right\|}$ and separation we get $c_{M_{H}} \leq C$, with $C$ independent of $A$ and $z_{j}$. From this it follows that

$$
\left\|k_{z_{n}}^{A_{n}}\right\| \geq C^{-1}\left\|k_{z_{n}}\right\|,\left\|k_{z_{m}}^{A_{m}}\right\| \geq C^{-1}\left\|k_{z_{m}}\right\|
$$

and

$$
\frac{k_{z_{m}}^{A^{\prime}}}{\left\|k_{z_{m}}^{A^{\prime}}\right\|^{2}}=\phi \frac{k_{z_{m}}}{\left\|k_{z_{m}}\right\|^{2}}
$$

where $\|\phi\|_{M_{H}} \leq C$. Thus

$$
\left|k_{z_{m}}^{A^{\prime}}\left(z_{n}\right)\right| \leq C\left|k_{z_{m}}\left(z_{n}\right)\right|
$$

and in (5) we get the estimate

$$
\left|\left\langle f_{n}, f_{m}\right\rangle\right| \leq C \frac{\left|k_{z_{m}}\left(z_{n}\right)\right|}{\left\|k_{z_{n}}\right\|\left\|k_{z_{m}}\right\|}
$$

Since $\Gamma$ is bounded we get from the assumption on the Gram matrix that $\Gamma^{\prime}$ is also bounded, which finishes the proof.

We remark that this proof works for any Hilbert space with a complete Nevanlinna-Pick kernel. This follows from the characterisation of such kernels in [1. Theorem 1.2].

We now prove that Theorem 1.1 applies to the Dirichlet space. The reproducing kernels are

$$
k_{w}(z)=\frac{1}{z \bar{w}} \log \left(\frac{1}{1-z \bar{w}}\right) .
$$

Assuming that the $\left\{a_{n}\right\}$ are positive, we need to bound

$$
\sum_{n, m} a_{n} a_{m} \frac{\left|k_{z_{m}}\left(z_{n}\right)\right|}{\left\|k_{z_{n}}\right\|\left\|k_{z_{m}}\right\|} .
$$

One checks that

$$
\left|\frac{1}{z \bar{w}} \log \left(\frac{1}{1-z \bar{w}}\right)\right| \lesssim C+\operatorname{Re} \frac{1}{z \bar{w}} \log \left(\frac{1}{1-z \bar{w}}\right)
$$

so that

$$
\sum_{n, m} a_{n} a_{m} \frac{\left|k_{z_{m}}\left(z_{n}\right)\right|}{\left\|k_{z_{n}}\right\|\left\|k_{z_{m}}\right\|} \lesssim\left(\sum_{n} \frac{a_{n}}{\left\|k_{z_{n}}\right\|}\right)^{2}+\operatorname{Re} \sum_{n, m} a_{n} a_{m} \frac{k_{z_{m}}\left(z_{n}\right)}{\left\|k_{z_{n}}\right\|\left\|k_{z_{m}}\right\|} .
$$

The second term is by assumption bounded by $\left\|a_{n}\right\|_{l_{2}}^{2}$. For the first term it follows from the Carleson measure condition that

$$
\left(\sum_{n} \frac{a_{n}}{\left\|k_{z_{n}}\right\|}\right)^{2} \leq\left\|a_{n}\right\|_{l_{2}}^{2} \cdot \sum_{n}\left\|k_{z_{n}}\right\|^{-2} \lesssim\left\|a_{n}\right\|_{l_{2}}^{2} .
$$


Similarly, Theorem 1.1 applies to the Dirichlet-type spaces $D_{\alpha}$ that lie between the Hardy and Dirichlet spaces. For these, the reproducing kernels are

It is clear that

$$
k_{w}(z)=\frac{1}{(1-z \bar{w})^{1-\alpha}}, 0<\alpha<1 .
$$

$$
\operatorname{Arg} \frac{1}{(1-z \bar{w})^{1-\alpha}} \in\left[-\frac{\pi}{2}(1-\alpha), \frac{\pi}{2}(1-\alpha)\right]
$$

which implies

$$
\frac{1}{|1-z \bar{w}|^{1-\alpha}} \leq C_{\alpha} \cdot \operatorname{Re} \frac{1}{(1-z \bar{w})^{1-\alpha}} .
$$

From this the conclusion follows easily.

Thus one gets a short proof of the theorem from [6] and 3] on interpolating sequences. We mention also [4, which gives a constructive proof that does not use the Nevanlinna-Pick property and applies to spaces defined with $L^{p}$-norms. For characterisations of Carleson measures for the Dirichlet space, we refer to [8].

The result on interpolating sequences for $D_{\alpha}$ is proved by different means in [5] and 10. We would like to point out that previous proofs for the Dirichlet space and the spaces $D_{\alpha}$ have been very different, whereas with the method here we obtain a unified treatment.

Theorem[1.1 is related to [2, Question 9.57], which asks if for a Hilbert space with a complete Nevanlinna-Pick kernel, a sequence being separated and having bounded Gram matrix must be an interpolating sequence. Theorem 1.1 gives an affirmative answer to this under the hypothesis on the Gram matrix. In this connection we point out that this extra hypothesis does not hold for the Hardy space $H^{2}$.

\section{ACKNOWLEDGMENT}

I would like to thank K. Seip for useful discussions.

\section{REFERENCES}

1. J. Agler and J. McCarthy, Complete Nevanlinna-Pick kernels, J. Funct. Anal 175 (2000). MR1774853 (2001h:47019)

2. vol. 44, American Mathematical Society, 2002. MR1882259 (2003b:47001)

3. C. Bishop, Interpolating sequences for the Dirichlet space and its multipliers, Preprint, 1994.

4. B. Boe, Interpolating Sequences for Besov Spaces, J. Funct. Anal 192 (2002), 319-341. MR1923404 (2003h:46044)

5. W. Cohn, Interpolation and multipliers on Besov and Sobolev spaces, Complex Variables 22 (1993), 35-45. MR:1277009 (95g:30069)

6. C. Sundberg and D. Marshall, Interpolating sequences for the multipliers of the Dirichlet space, see www.math.washington.edu/ marshall/preprints/preprints.html.

7. N.K. Nikolskii, Treatise on the Shift Operator, Springer-Verlag, 1980. MF0827223 (87i:47042)

8. R. Rochberg, E. Sawyer, and N. Arcozzi, Carleson measures for analytic Besov spaces, Rev. Mat. Iberoamericana 18 (2002), no. 2, 443-510. MR1949836 (2003j:30080)

9. H. Shapiro and A. Shields, On the zeros of functions with finite Dirichlet integral and some related function spaces, Math. Z. 80 (1962). MR0145082(26:2617)

10. J. Xiao, The delta-bar problem for multipliers of the Sobolev spaces, Manuscripta Math. 97 (1998), 217-232. MR.1651405 (99g:46047)

Department of Mathematics, University of California los Angeles, Box 951555, Los Angeles, California 90095-1555

E-mail address: bjarteb@math.ucla.edu 